Retos, $n^{\circ} \mid 4$, vol.VIII, 2017

\title{
Microempresas panificadoras en el Azuay (Ecuador) y su productividad
}

\author{
Bakery small business in Azuay (Ecuador) \\ and their productivity
}

\begin{abstract}
Carlos Armando Romero Galarza es investigador del Grupo de Investigación Empresarial (GIE) en la Facultad de Ciencias Económicas y Administrativas de la Universidad de Cuenca (Ecuador) (armando.romerog@ ucuenca.edu.ec) (http://orcid.org/0000-0003-1149-4299)
\end{abstract}

Gustavo Geovvani Flores Sánchez es Docente de la Universidad de Cuenca e investigador del Grupo de Investigación Empresarial (GIE) en la Facultad de Ciencias Económicas y Administrativas de la Universidad de Cuenca (Ecuador) (gustavo.flores@ucuenca.edu.ec) (http://orcid.org/0000-0003-4123-2644)

\begin{abstract}
Jorge Arturo Campoverde Campoverde es Docente Titular de la Universidad de Cuenca en la cátedra de Investigación de Operaciones e investigador y coordinador del Grupo de Investigación Empresarial (GIE) en la Facultad de Ciencias Económicas y Administrativas de la Universidad de Cuenca (Ecuador) de la Universidad de Cuenca (jorge.campoverde@ucuenca.edu.ec) (http://orcid.org/0000-0002-1633-5644)
\end{abstract}

Katherine Tatiana Coronel Pangol es egresada de la carrera de Administración de Empresas y Ayudante de Investigación del Grupo de Investigación (GIE) en la Facultad de Ciencias Económicas y Administrativas de la Universidad de Cuenca (Ecuador) (katherine.coronelp95@ucuenca.ec) (http://orcid.org/0000-0003-4370-756X)

\section{Resumen}

El sector empresarial representa uno de los ejes fundamentales en la economía de un país, en la generación de empleo y en el desarrollo del sector productivo, comercial y de servicios. Es notoria la participación que tiene la micro, pequeña y mediana empresa debido a la gran cantidad de organizaciones existentes en esta categoría. Cabe recalcar que es preocupante el corto ciclo de vida que mantienen las mismas y las limitantes que éstas mantienen. El estudio presenta un análisis descriptivo y bivariado de 113 microempresas panificadoras en la provincia del Azuay (Ecuador), afiliadas al Ministerio de Industrias y Productividad (MIPRO) de la Zona 6. Los resultados muestran comportamientos relacionados entre el promedio de productividad por empleado que tienen las microempresas y la gestión empresarial estratégica, la capacidad productiva, el grado de innovación en maquinaria, equipo y sistemas de comercialización y sobre el requerimiento de financiamiento adicional para el crecimiento de los negocios. Se considera que es un sector con limitada mano de obra calificada que no le permite un incremento en la productividad y competitividad, factor clave de desarrollo empresarial.

\begin{abstract}
The business sector represents one of the fundamental axes in the economy of a country. It contributes to generate employment; development of the industrial, commercial and services sector. At the same time, the participation of micro, small and medium-sized enterprises is noteworthy due to the large number of organizations in this category. It is important to emphasize in the short life cycle of these companies and the limitations that are present. Through a descriptive and a bivariate analysis, 113 micro-bakeries had been studied in the province of Azuay, which are affiliated to the Ministry of Industries and Productivity (MIPRO) of Region 6. The results show related behaviors between the average productivity per employee of the microenterprises and strategic business management, their productive capacity, their degree of innovation in machinery, the equipment and marketing systems and also on the requirement of additional financing for the growth of business. However, it is considered that it is a sector with limited skilled labor that does not allow an increase in productivity and competitiveness, which is a key factor in business development.
\end{abstract}

\section{Palabras clave | keywords}

Microempresas, productividad, innovación, gestión empresarial, estrategia, financiamiento. Microenterprise, productivity, innovation, business management, strategic, financing. 


\section{Introducción}

Es indiscutible la importancia de la micro y pequeña empresa hoy en día en la economía nacional e internacional. De acuerdo a cifras arrojadas por el INEC - Directorio de Empresas y Establecimientos (2014), en el Ecuador representan el $97.9 \%$ del total de las organizaciones productivas.

El sector manufacturero es uno de los más importantes debido a los grandes encadenamientos que genera en la cadena de valor, generando empleo e innovación. La estructura de encadenamientos en una economía es irrelevante desde el punto de vista neoclásico más ortodoxo, no obstante, otros economistas que dan mayor preponderancia a los hechos estilizados como Kuznets (1966) y Rodríguez, Bernal, \& Cuervo (2012) han sugerido que el crecimiento económico es impulsado por los encadenamientos productivos entre diferentes tamaños de empresas que se dan en los territorios.

Al contrario, América Latina y en especial la de Ecuador, se caracteriza por la presencia de una realidad empresarial fuertemente polarizada y desarticulada "...en la cual conviven polos altamente competitivos con universos dispersos de micro, pequeñas y medianas empresas (Mipymes) escasamente desarrolladas" (Cohen \& Gabriel, 2012).

Las Pymes bien administradas y saludables son fuentes de empleo y creación de riqueza. Contribuyen a la estabilidad social y generar ingresos fiscales. Según la Corporación Financiera Internacional (2009) existe una relación positiva entre el nivel de ingresos de un país y el número de Pymes por cada 1000 personas. Los informes del Doing Business (World Bank Group, 2014) indican que un sector alto de Pymes corresponden a un nivel reducido de actividades en el sector informal o mercado negro.

Por otro lado, en América Latina el impulso a la actividad emprendedora se ha convertido en una de sus principales políticas, considerando a las microempresas y las pequeñas empresas con una representatividad del $80 \%$ del total de la economía (Hidalgo, Kamiya, \& Reyes, 2014). En el Ecuador, de acuerdo al informe trimestral del mercado laboral del Banco Central del Ecuador (2015), se observó que la mayoría de emprendedores se encuentran en establecimientos con menos de 100 empleados Mypimes; este porcentaje se ubica en el 77,4\%; mientras que el 22,6\% restante se ubican en establecimientos con más 
de 100 empleados; es decir, se evidencia un rol importante de las micro y pequeñas empresas en la economía ecuatoriana.

Sin embargo, de acuerdo a Cohen \& Gabriel (2012) las Mypimes tienen algunas dificultades como es el acceso restringido a las fuentes de financiamiento, bajos niveles de capacitación de sus recursos humanos, limitados niveles de innovación y desarrollo tecnológico, poca penetración en mercados internacionales, bajos niveles de productividad, baja capacidad de asociación y administrativa entre los más importantes; factores que limitan su crecimiento empresarial, restando su productividad y eficiencia en los mercados nacional como internacional.

El objetivo de la presente investigación es evidenciar si los factores referidos ut supra afectan la productividad de los empleados de las microempresas panificadoras con la gestión empresarial desarrollada y en qué forma, mediante la influencia de ciertas variables relacionales que forman parte de la organización de los negocios. Las variables relacionales que se incluyen en el análisis están influenciadas con determinadas estructuras de gestión empresarial como son de tipo estratégico, operativo, además de factores de innovación y requerimiento de financiamiento adicional para las operaciones de las microempresas panificadoras.

\section{Marco teórico}

\subsection{Desempeño organizacional}

Para entender el concepto de desempeño organizacional resulta necesario aclarar acepciones que pueden confundirse con la de efectividad organizacional que hace referencia al cumplimiento de objetivos propuestos y la de desempeño de la firma que se constituye en el valor de mercado.

Según Dyer \& Reeves (1995), el desempeño organizacional hace referencia a la medición y evaluación de la obtención de objetivos de la organización y se miden a través de la evaluación de resultados financieros, resultados organizacionales y resultados relacionados con recursos humanos.

Velarde, Araiza, \& García (2014) realizan un estudio en Coahuila, México, en el que tratan de determinar la relación entre los factores de la empresa y el empresario con el éxito empresarial, y concluyen que tan sólo el factor asociado al grado de escolaridad tiene una relación positiva con el crecimiento en la inversión de maquinaria y equipo, mientras que 
el resto de factores de los empresarios no tienen asociación con el éxito económico. Por su parte, los factores de la empresa -tales como estructura jurídica y sector económico en el que se desarrolla la empresa- tienen relación con el éxito de la empresa en lo concerniente a incremento de ventas, incremento de empleados e inversión en maquinaria y equipo.

\subsection{Innovación}

En la actualidad, de acuerdo a Teece, Pisano, \& Shuen (1997), el crecimiento en las organizaciones y en especial las MIPYMES manufactureras requieren entre otras opciones, fortalecer su actividad innovadora y para ello, requieren tener una mayor competitividad. Lochon (2014) considera en sus trabajos de investigación, que la actividad innovadora debe tener una influencia en las empresas de tal manera que ésta debe verse reflejada en los resultados empresariales y en el incremento gradual de su competitividad.

Un modelo de análisis ampliamente reconocido por la literatura asume que la realización de innovaciones por parte de las empresas está influida por un conjunto de variables, las cuales pueden agruparse básicamente en tres grandes bloques como describen Hoffman, Parejo, Bessant, \& Perren (1998), entre las que se cuentan: i) factores extra-organizativos, en particular asociados a características del sector al que pertenece la empresa, ii) las vinculadas a las características de los miembros de la organización, en especial los directivos, iii) las relativas a la propia organización, por ejemplo la orientación estratégica o la estructura financiera.

Según Etzkowitz \& Leydesdorff (2000) la innovación se ha convertido en uno de los elementos clave dentro de una empresa. Ésta trasciende a todas las áreas como lo son producción, finanzas, planificación, gestión de recursos humanos y marketing. Sin embargo, en la pequeña empresa muchas de estas funciones son llevadas a cabo directamente por el propietario o por responsable que ejecuta varios trabajos.

Las pequeñas empresas tienen enormes ventajas en materia de innovación respecto a las grandes empresas como su dinamismo empresarial, su flexibilidad interna y su capacidad de respuesta a las circunstancias cambiantes, además por su tamaño les aporta dinamismo, flexibilidad interna, cortos plazos de entrega de los productos y capacidad de respuesta al cambio, aunque pueden encontrarse con barreras como: 
- Falta de especialistas y personal calificado dentro de las pequeñas empresas.

- Carencia de tiempo y recursos para identificar y utilizar fuentes externas de información, técnicas y conocimientos científicos.

- Dificultades para atraer capital, especialmente capital riesgo

- Incapacidad de la pequeña empresa para distribuir el riesgo a través de una cartera de proyectos debido a sus recursos limitados.

Sin embargo Vossen (1998) afirma que cierta corriente de la literatura considera que existen diferencias entre grandes y pequeñas empresas. Esto justifica que la actividad innovadora de estas últimas sea más desarrollada internamente y esté mayormente vinculada con la figura del empresario y la toma de ciertas estrategias empresariales. Algunos estudios respaldan que las pequeñas empresas suelen ser más exitosas en industrias donde el peso de las habilidades y capacidades personales son más importantes, mientras que otros afirman que las fortalezas de la pequeña empresa no residen en los recursos sino en características como la flexibilidad, la cultura empresarial y la motivación al personal.

Varios autores dan a conocer la importancia que tiene la inversión en innovación para el incremento del nivel de ventas en las micro y pequeñas empresas, así por ejemplo Benavente (2005) determinó que un mayor gasto en Investigación y Desarrollo (I+D), conformado por investigación de las ciencias y el desarrollo tecnológico, está positivamente relacionado al aumento de la importancia que tienen la ventas de productos innovadores sobre las ventas totales de la empresa.

Analizando una muestra de empresas manufactureras chilenas, Griliches (1979) presenta como resultados de su investigación una relación positiva, entre gastos en I+D y el aumento de la productividad. El vínculo existente entre inversión en innovación es también evidenciado por Crépon et al. (1988) y Kemp et al. (2003), aunque cabe recalcar que también existen estudios que indican una no relación consistente entre las mismas variables (Lööf, Heshmati, Asplund, \& Naas, 2001).

Asimismo Córdova \& Naranjo (2017), manifiestan que las empresas pequeñas con menor nivel tecnológico realizan un mayor esfuerzo en actividades de Ciencia Tecnología e Investigación, lo cual no se ve reflejado en ventas innovadoras, mientras que las empresas con mayor nivel de tecnificación superan en niveles de ventas a las anteriores. 
Por otro lado Fairlie (2007), buscó determinar si existe una relación estadística entre la innovación empresarial y la generación de valor agregado, donde las comparaciones realizadas en general demostraron que un $82 \%$ de las PYMES que han aplicado algún tipo de innovación en su empresa en los últimos dos años han visto incrementadas sus ventas en relación a sus competidores, mientras que aquellas que no lo hicieron han tenido un incremento de ventas del 27\%. Las diferencias en ventas entre empresas del mismo rubro con respecto a aquellas que se innovan constantemente es significativa.

\section{Gráfico 1. Funciones de la administración}

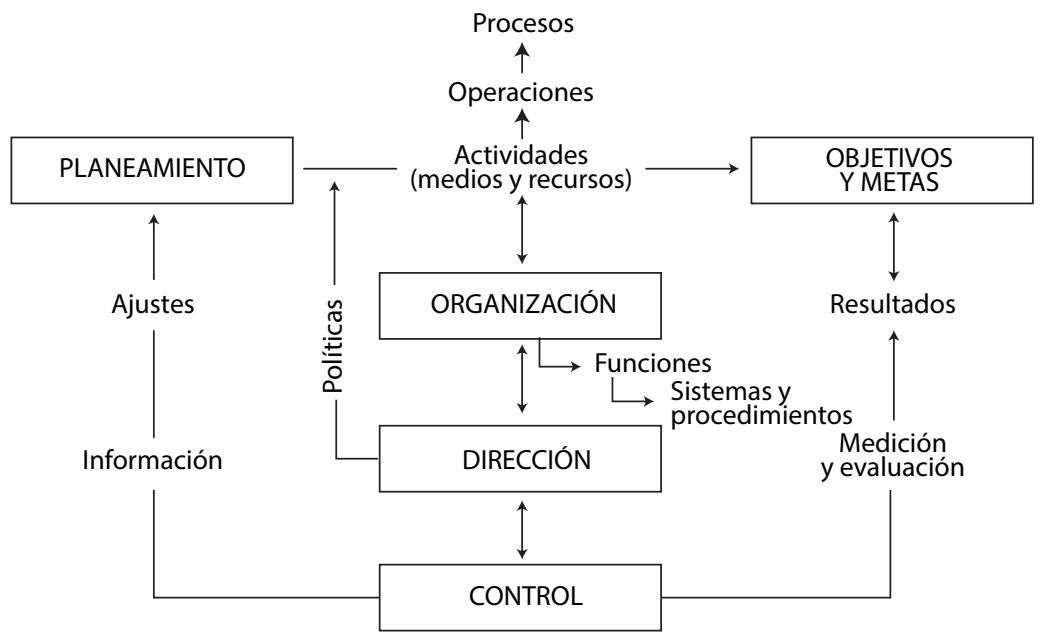

Fuente: Dextre (2010)

\subsection{Rendimiento de las MIPYMES}

Considerando que la administración diseña la estructura sobre la que se soporta la función de control de las empresas, para Solís (2005), el diseño de los sistemas de control debe ofrecer una retroinformación en forma oportuna y barata, que sea aceptable para los miembros de la organización. A partir de ello, se puede afirmar que el sistema de control basado en la estructura de la organización desarrolla las actividades del giro del negocio, bajo políticas claras y definidas orientadas a preservar el fin común. 
La administración tiene como funciones fundamentales el planeamiento, la organización, la dirección y control. La coherencia y cohesión entre ellas produce una administración eficiente, económica y efectiva de los recursos que emplea para el logro de las metas y objetivos planeados. En tal sentido, se definen las funciones de la administración para centrar el análisis en el control y su vinculación con los riesgos de negocios. En el gráfico 1 se puede observar el proceso que sigue el desarrollo de las funciones dentro de la administración.

\section{Materiales y métodos}

\subsection{Tipo de investigación}

La investigación es de tipo experimental, ya que se centra en la puesta en práctica de los conocimientos para la búsqueda del beneficio social, mediante la utilización y estudio de variables que permitirán entender el comportamiento del sector.

\subsection{Método de investigación}

El método de investigación aplicado es el hipotético-deductivo, debido a que permite hacer demostraciones en base a las hipótesis o preguntas de investigación; a su vez también se utilizó el método lógico-deductivo, debido a que admite considerar muestras poblacionales para la estimación de resultados ya que los elementos de la investigación no pudieron ser estudiados en su totalidad.

\subsection{Muestra}

Para la determinar la muestra se consideró como población de estudio a las micro y pequeñas empresas del sector panificador registradas en la base de datos del Ministerio de Industrias y Productividad (MIPRO) en la provincia del Azuay (Ecuador) de enero 2015 a marzo 2016, considerando un total de 137 panaderías; por lo que el levantamiento de información se realizó de manera censal.

De las 137 panaderías, se logró encuestar a 113, lo cual representa un $82,5 \%$ de la población. Del 17,5\% restante, es decir 24 negocios, no se obtuvo respuesta por diversos motivos: el 2,92\% vendió el negocio, el $1,46 \%$ salió de viaje - vacaciones, el 10,20\% no se ubica debido a que 
tanto la dirección como el teléfono están erróneos, un 0,73\% no autoriza, el 1,46\% no colabora, y el 0,73\% cambio de negocio.

En la tabla 1, se da a conocer cómo se determinó el margen de error muestral para poder inferir en toda la población resultante, obteniendo un $+/-3,88$ margen suficiente que permite inferir en toda la población de estudio.

Tabla 1. Parámetros estadísticos muéstrales

\begin{tabular}{|l|l|}
\hline \multicolumn{1}{|c|}{ Descripción } & \multicolumn{1}{c|}{ Valor } \\
\hline Nivel de confianza & $95 \%$ \\
\hline Población & 137 \\
\hline Margen de error muestral & $+/-3,88$ \\
\hline Muestra & 113 \\
\hline
\end{tabular}

\subsection{Fuentes de información}

La información recopilada para la elaboración de la investigación se obtiene mediante información primaria aplicando encuestas a cada una de las microempresas del sector en estudio. También se obtuvo información secundaria que se fundamentó en la revisión de bibliografía de interés. Finalmente se consiguió información de fuentes terciarias que brindaron información a nivel macroeconómico del sector de estudio, tales como el Instituto Nacional de Estadísticas y Censos (INEC).

\subsection{Procedimiento metodológico y herramientas}

El proceso llevado a cabo en el estudio inicia con la obtención de la base de datos de las micro y pequeñas empresas a ser investigadas. Posteriormente se procedió con la recolección de datos en fuentes primarias; luego se elaboraron las herramientas para levantamiento y análisis de información, tales como instrumento-encuesta y plantillas para generación de bases de datos, mismas que fueron puestas a prueba en un análisis exploratorio piloto que sirvió para validar y garantizar la fiabilidad de las mismas.

Las encuestas se aplicaron de manera directa a cada uno de los propietarios y/o administradores de las empresas, por lo que se garantiza la veracidad de los datos. Una vez concluida la aplicación de las encuestas se procedió a verificar el llenado de la información e ingresar los datos en las plantillas del software de análisis. 
Una vez digitalizados los datos de las encuestas fueron procesados en el software estadístico IBM SPSS® v. 20.0, mediante un análisis de información estadística univariado (frecuencias y porcentajes) de las principales variables de estudio. Esto permitió caracterizar al sector panificador en la provincia del Azuay, indagando sus oportunidades y debilidades. Además se realizó un análisis bivariado para contrastar las diferencias entre las medias de más de dos poblaciones. Para el efecto, se utilizó ANOVA para determinar y comparar entre sí las medias de la productividad por empleado de las microempresas con factores relevantes de la gestión empresarial que inciden en su operación manufacturera.

\subsection{Descripción de datos y variables}

Para evaluar a las microempresas panificadoras, se estableció un conjunto de variables consideradas según realidad del sector. Éstas permitieron tener una visión general de la situación. Las variables definidas fueron:

- Variable dependiente: se considera el promedio de productividad del empleado, medido por la facturación mes sobre el número de empleados que disponen las microempresas panificadoras.

- Variables independientes:

$\sqrt{ }$ Gestión Empresarial: definida su misión.

$\sqrt{ }$ Gestión Empresarial: definida su visión.

$\sqrt{ }$ Gestión Empresarial: definido su organigrama.

$\sqrt{ }$ Gestión Empresarial: definidas las políticas internas de la empresa.

$\sqrt{ }$ Capacidad Productiva: número de unidades/día producidas.

$\sqrt{ }$ Cambio o mejora en sus procesos, productos o sistemas gestión en los últimos 2 años.

$\sqrt{ }$ Innovación realizada: Adquisición de tecnología incorporada a maquinaria y equipo.

$\sqrt{ }$ Innovación realizada: Incorporado mejoramiento de los sistemas de comercialización.

$\sqrt{ }$ Financiamiento adicional durante el tiempo que lleva generando actividades.

\subsection{Promedio de Productividad del empleado}

El desempeño laboral es la forma en que los empleados realizan su trabajo. Este se evalúa durante las revisiones de su rendimiento, mediante 
el análisis de factores como la capacidad de liderazgo, la gestión del tiempo, las habilidades organizativas y la productividad para analizar cada empleado de forma individual (Comisión Económica para América Latina y el Caribe - CEPAL, 2001).

Las revisiones del rendimiento laboral, por lo general, se llevan a cabo mensual o anualmente y pueden determinar que se eleve la elegibilidad de un empleado, decidir si es apto para ser promovido o incluso si debiera ser despedido. Por lo tanto, el rendimiento está ligado a la productividad de los empleados, el cual se mide a través del cociente entre la facturación de una empresa y el número de empleados que tiene. El número de empleados, por su parte, es una variable que también indica el tamaño de gran dimensión que tienen las empresas (Bonilla $\&$ Mayorga, 2011).

La productividad de los empleados es consecuencia del rendimiento laboral, que es la relación entre objetivos, metas o tareas alcanzadas y el tiempo que se ha necesitado para lograrlo. Este cálculo ha de realizarse teniendo en cuenta que el tiempo se refiere a las horas trabajadas de calidad y que la variable más importante son las personas encargadas de ejecutar las funciones propias del puesto de trabajo (Rodríguez \& Gómez, 2011).

\subsection{Ventas y productividad general}

De acuerdo a Salazar (2015), el número promedio de ventas que generan los empleados es una buena medida de la productividad, ya que ayuda a determinar en cuánto deben aumentar las ventas para justificar la contratación de un empleado adicional, por lo tanto para obtener un promedio de productividad de los empleados, se deriva la siguiente ecuación:

Promedio de Productividad de los empleados $=\frac{\text { ventas netas }}{\text { Número de empleados }}$

El promedio de productividad de los empleados describe en cuánto debe aumentar las ventas para considerar el costo de contratar a un nuevo miembro del personal en la empresa. Evaluar el desempeño o productividad de los empleados puede proporcionar numerosos beneficios para la organización. Además le permite distribuir de forma equitativa los incentivos de compensación. Las evaluaciones de desempeño de los 
empleados pueden aumentar la eficiencia operativa y la productividad del personal, al mismo tiempo que identifica a los candidatos de alto rendimiento para una promoción futura en las empresas (MaldonadoGuzmán et al., 2011).

Igualmente en la tabla 2 se aprecian los estadísticos descriptivos de la variable de interés, que es el promedio de la productividad de los empleados/mes de las microempresas panificadoras, cuyo valor es de USD 683,28

\section{Tabla 2. Estadístico Descriptivo}

\begin{tabular}{|c|c|l|l|l|l|c|}
\hline Variable & No. & Mínimo & Máximo & Media & Mediana & $\begin{array}{c}\text { Desv. } \\
\text { Típica }\end{array}$ \\
\hline Productividad por empleados & 113 & 20 & 2,400 & 683,28 & 500 & 536,48 \\
\hline
\end{tabular}

Nota: Elaboración propia con el software SPSS $®$ v.20.0

\section{Descripción del sector}

En Ecuador existen 6.879 empresas activas dedicadas a la elaboración de productos de panadería, según el calificador de actividades económicas CIIU4 - C1071. De este total, dentro de la provincia del Azuay se encuentran un total de 621 empresas, de las cuales una representación del $83,89 \%$ se encuentra en el cantón Cuenca. A su vez, las micro y pequeñas empresas representa un $67,99 \%$ del total de empresas ecuatorianas (INEC 2015, Redatam). Su participación en distintos escenarios es muy heterogéneo, siendo el más importante en la generación de empleo, menos importante en la producción y muy pequeña en las exportaciones.

La mayor participación en el empleo, comparada con la producción, indica bajos niveles relativos de productividad. Su escasa participación en las exportaciones muestra su fuerte orientación al mercado interno y su dependencia de la dinámica de la demanda interna. Por ello, su producción está muy determinada por la evolución del empleo y salarios en la economía en su conjunto, contribuyendo de esta manera con el 0,28\% del empleo formal según datos (INEC 2015). La tabla 3, presenta el crecimiento que ha tenido el sector en su nivel de ventas. 
Tabla 3. Variación porcentual de ventas anuales

\begin{tabular}{|l|c|c|}
\hline \multicolumn{1}{|c|}{ Periodo } & $\begin{array}{c}\text { Ventas } \\
\text { (miles de millones de USD) }\end{array}$ & Variación de ventas \\
\hline Año 2011 & $\$ 4430,54$ & $0,00 \%$ \\
\hline Año 2012 & $\$ 4821,58$ & $8,83 \%$ \\
\hline Año 2013 & $\$ 5462,98$ & $13,30 \%$ \\
\hline Año 2014 & $\$ 7340,94$ & $34,38 \%$ \\
\hline Año 2015 & $\$ 7856,61$ & $7,02 \%$ \\
\hline Año 2016 & $\$ 8794,00$ & $11,93 \%$ \\
\hline Año 2017 & $\$ 9731,15$ & $10,66 \%$ \\
\hline Promedio & $\$ 6919,68$ & $14,35 \%$ \\
\hline
\end{tabular}

Fuente: Elaboración propia con datos del INEC (2011 - 2015)

En el periodo 2011-2017 el sector panificador ha mostrado tener un crecimiento constate, mismo que alcanza un promedio de aproximadamente $14,35 \%$. El gráfico 2 muestra la tendencia de crecimiento que ha tenido el nivel de ventas del sector, considerándose un rubro con crecimiento importante en la economía del Ecuador. Las ventas del 2016 y 2017 fueron pronosticadas.

\section{Gráfico 2. Ventas anuales sector panificador}

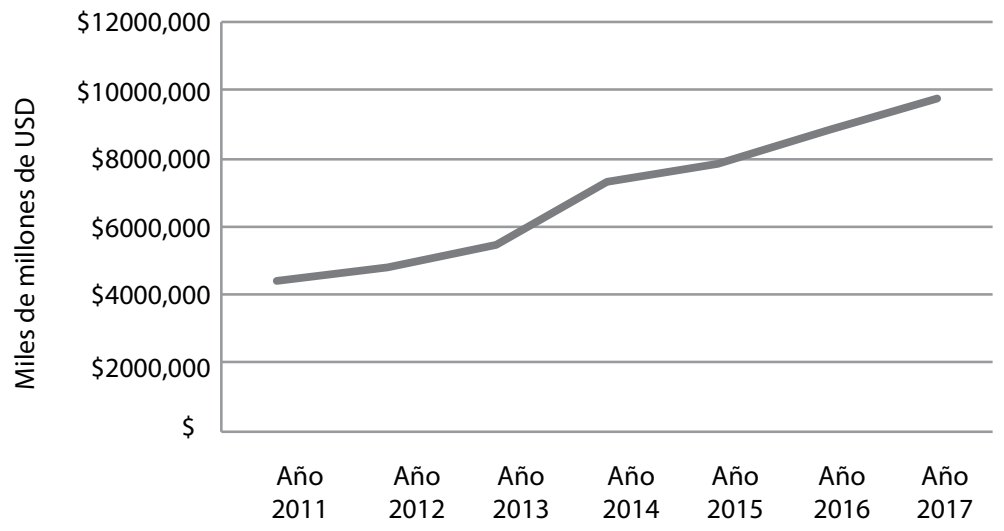

Fuente: Elaboración propia con datos el INEC (201 1-2015) 


\section{Análisis y resultados}

Los resultados obtenidos mediante el análisis descriptivo de las 113 encuestas aplicadas remiten que el 90\% de encuestados están calificados como artesanos afiliados al MIPRO, mientras que el 10\% pertenecen a la Junta del Artesano. De estos, el $89 \%$ no tiene la obligación de llevar contabilidad ya que están calificados bajo la modalidad Régimen Impositivo Simplificado Ecuatoriano (RISE) y tan solo un 11\% tiene la obligación de llevar contabilidad.

Considerando los años de la actividad de las empresas, se tiene que el 44\% mantienen sus actividades entre 1 y 5 años; el 24\% llevan entre 5 y 10 años dedicándose; el 13\% entre 10 y 15 años y el 19\% cumple con sus actividades por más de 15 años.

En la gráfico 3 se observa con meridiana claridad que alrededor del 20\% de los negocios encuestados cuentan con 1 empleado, el 51\% de estos cuentan con 2 empleados, el 17\% disponen de 3 empleados, el $11 \%$ de los negocios disponen de 4 empleados, y solo el 2\% tiene más de 5 empleados; evidenciando que el $99 \%$ de las empresas por su característica son microempresas.

\section{Gráfico 3. Número de empleados que tienen las empresas panificadoras}

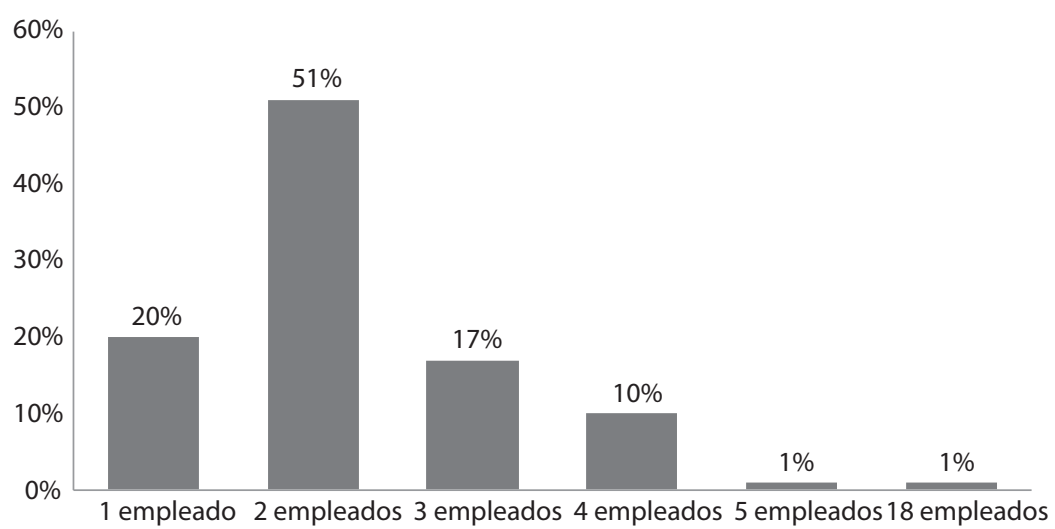

En cuanto al mercado de destino de sus ventas, se observa que el 96\% de su facturación se concentran en la ciudad de Cuenca y el 4\% lo realiza en cantones y provincias cercanas. Por otro lado, en lo que respecta al giro del 
negocio, se tiene que el $93 \%$ de las microempresas producen pan y pastelería, mientras que el 7\% producen solo productos de pastelería.

La facturación mensual promedio de los 113 negocios muestra una media de ingresos mensuales de $\$ 1708,32$. Además se evidencia que existen en promedio 5 locales de la misma actividad económica en un radio de 5 cuadras, considerándolo como un negocio con muchos competidores.

Se observa que los empleados de las microempresas tienen en promedio 8 años de escolaridad; un 43\% de los empleados tienen educación primaria, el 51\% de los empleados tienen educación secundaria, el 5\% tienen una formación universitaria y el restante $1 \%$ tienen un nivel de posgrado.

De igual manera, es relevante mencionar que el $58 \%$ de negocios han recibido capacitación, además que un $59 \%$ de las microempresas que han recibido capacitación lo han realizado en temas como el manejo administrativo y facturación, un $24 \%$ en manejo de proveedores y un $12 \%$ en ventas y atención al cliente, según se indica en el gráfico 4.

\section{Gráfico 4. Áreas de capacitación recibida por los empleados de las empresas panificadoras}

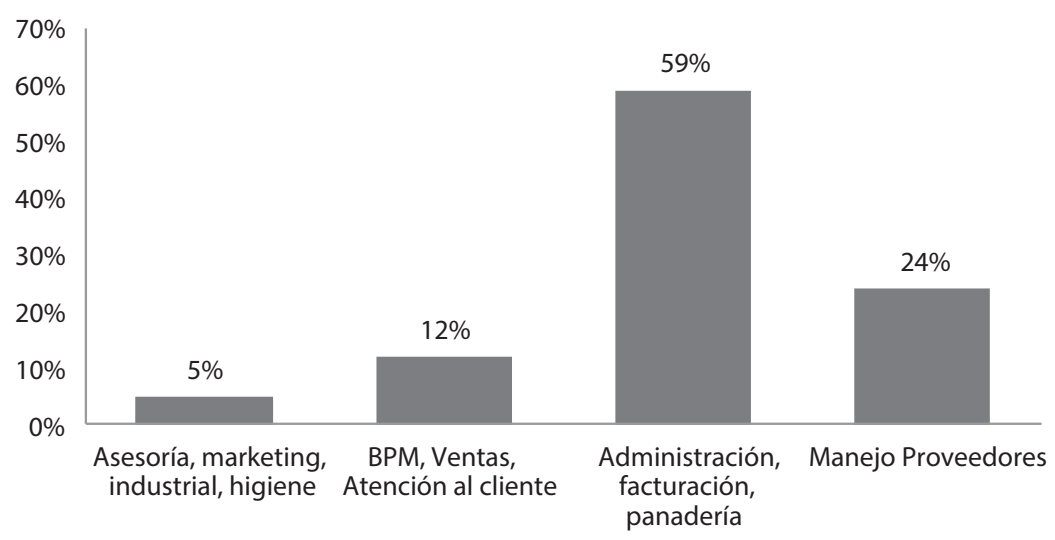

Por otra parte se establece que durante el tiempo que llevan operando los negocios, solo el $43 \%$ de las empresas ha solicitado financiamiento adicional para continuar con sus operaciones, siendo su monto promedio de financiamiento de $\$ 12885,45$. En el gráfico 5 se observa el motivo por el cual requieren financiamiento adicional; un $52 \%$ ha utilizado para la compra de maquinaria, un $30 \%$ para la ampliación física 
del negocio y un 24\% para adquisición de capital de trabajo como es la materia prima, entre los más importantes.

Gráfico 5. Motivo por el cual se requirió financiamiento

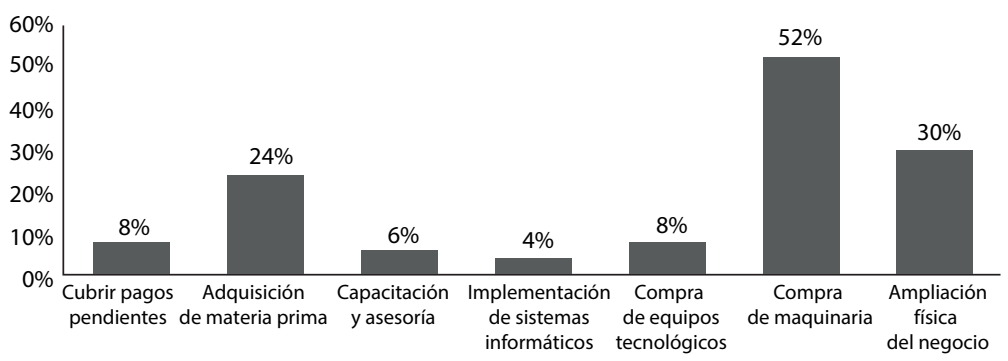

En lo que respecta a tecnología, maquinaria, y al grado de automatización de las microempresas, se aprecia que el 47\% de los negocios tienen una acción manual, es decir que la producción de pan y sus derivados es artesanal, mientras que un 47\% tienen una acción semi-automática y tan solo el $6 \%$ de los negocios tiene un grado automático. El 94\% de las empresas no disponen de programas informáticos para sus procesos o gestión.

Al examinar la relación entre los productos y procesos, definido por el tipo de proceso productivo que disponen las microempresas, se observa que el $71 \%$ lo hacen de una forma continua para la venta, el $19 \%$ produce para stock y solo el 10\% lo hace bajo pedido. Además, el $70 \%$ de las microempresas tienen una capacidad productiva utilizada de más de un $61 \%$, produciendo en promedio al día 658 unidades.

Al mismo tiempo, el 91\% de las empresas panificadoras proyecta su producción diariamente y solo el $9 \%$ lo realiza semanalmente. De igual manera los negocios fabrican un mix de productos, esto quiere decir que un $81 \%$ de las microempresas elaboran en promedio entre 1 y 5 productos, y un $19 \%$ producen más de 6 productos.

En lo que respecta al sistema de calidad que poseen los negocios, se puede apreciar que el $92 \%$ de las microempresas realizan controles de calidad de sus productos terminados, utilizando principalmente el método de inspección visual con un 92\%. Por otro lado, solo un 50\% de las empresas realizan controles de calidad en los procesos productivos; además el 95\% de las empresas no disponen de registros de control de 
calidad. De igual manera, el $70 \%$ de las empresas poseen un plan de mantenimiento de sus maquinarias y equipos, realizando un $46 \%$ mantenimiento correctivo y un $54 \%$ preventivo.

En lo pertinente al conocimiento y gustos del cliente, un 95\% de las empresas afirman conocer el gusto de los consumidores, además el 80\% conocen el hábito de compra de sus clientes. Por otro lado, solo el $43 \%$ identifica los productos y los precios de su competencia; el $26 \%$ presta servicios post venta y el $85 \%$ ofrece garantía en sus productos a sus clientes.

Si se analiza el origen y provisión de las materia prima, se obtiene que un $90 \%$ de las empresas se abastecen principalmente a nivel nacional, adquiriendo a distribuidores mayoristas. Además, el 50\% de las microempresas califican a sus proveedores y un $87 \%$ considera que el aprovisionamiento o la entrega es oportuna. De igual manera el $90 \%$ de las empresas manifiestan que el crédito concedido por sus proveedores es en promedio de 1 a 30 días.

En lo concerniente a la organización y gestión empresarial, se observa que el $92 \%$ de las empresas panificadoras no disponen de una gestión estratégica documentada. Por otro lado, el 78\% de las microempresas no llevan contabilidad general, ni contabilidad de costos; factores importantes para la toma de decisiones de los negocios. De igual manera el $80 \%$ de las empresas no usan planeación financiera como presupuesto de ventas, de compras, de producción y flujos de cajas para las operaciones del negocio.

\section{Gráfico 6. Principales limitantes para el crecimiento de la empresa}

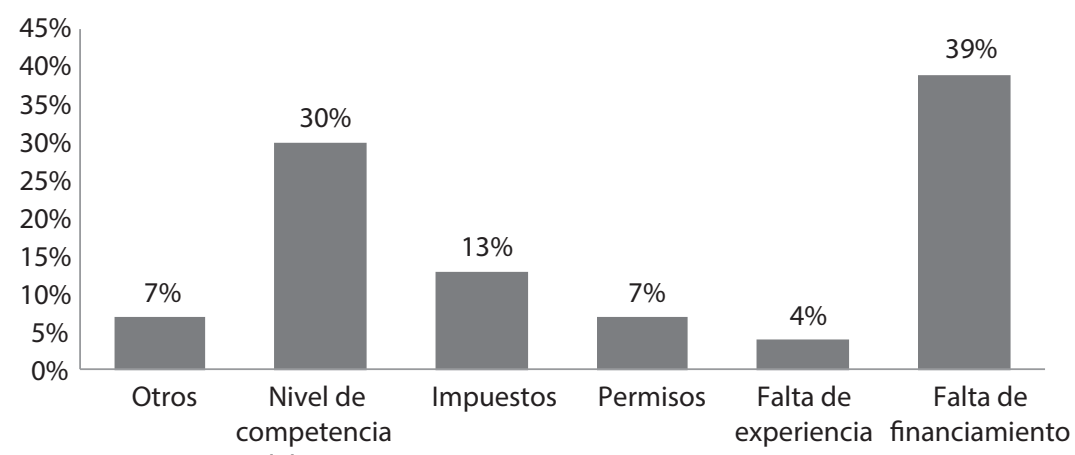


También fueron indagados acerca de las principales limitantes que tienen las microempresas para el crecimiento empresarial y según muestra el gráfico 6, el 39\% manifestó como causa la falta de financiamiento, el 30\%, el nivel de competencia del sector y el 13\% la carga impositiva de los impuestos, entre los más relevantes.

De igual forma, en lo que respecta a la percepción en la gestión en innovación y desarrollo por parte de las empresas, se obtiene que un 94\% consideran que la innovación es importante y muy importante para el desarrollo y crecimiento de las empresas. Inclusive un $62 \%$ de los negocios afirma haber realizado cambios en sus productos, procesos o sistemas de gestión en los últimos 2 años. De igual manera un $86 \%$ de las empresas han realizado innovación en el mejoramiento de los productos; además un $82 \%$ en mejoramiento en los procesos, un $51 \%$ han desarrollado nuevos productos y por último un $46 \%$ de los negocios han implementado normas ambientales y aplicación de tecnologías limpias en sus procesos productivos.

Posteriormente, se analizan las herramientas y equipo que disponen las microempresas, por lo que se observa que un $78 \%$ de las mismas utilizan mesas de trabajo de acero inoxidable, un $43 \%$ usan horno automático, el 53\% emplean horno manual, el $81 \%$ dispone de batidora, y el 59\% utiliza amasadora semi-automática, entre el equipamiento panadero más importante.

En lo relacionado, al análisis bivariado se realiza un análisis de varianza ANOVA de un factor (también llamada ANOVA unifactorial o one-way ANOVA), siendo una técnica estadística que establece si dos variables (una independiente y otra dependiente) están relacionadas en base a que si las medias de la variable dependiente son diferentes en las categorías o grupos de la variable independiente. Es decir, señala si las medias entre dos o más grupos son similares o diferentes.

Para el efecto, en la tabla 4, se presentan los resultados de contraste del análisis de ANOVA de la media de la variable de interés que es la productividad del empleado que tienen las microempresas panificadoras y las medias de las dos muestras independientes o factores concernientes a la gestión empresarial estratégica, operación de las empresas, aplicaciones de innovaciones y requerimiento de financiamiento adicional. Este análisis se generó de manera individual entre cada variable independiente con la variable dependiente. 


\section{Tabla 4. Análisis ANOVA de un factor-Desempeño del empleado / variables independientes}

\begin{tabular}{|c|c|c|c|}
\hline \multirow{2}{*}{\multicolumn{2}{|c|}{ Variables independientes (factor) }} & \multirow[t]{2}{*}{$\mathrm{N}$} & \multirow{2}{*}{$\begin{array}{c}\text { Desempeño } \\
\mathrm{F}\end{array}$} \\
\hline & & & \\
\hline \multirow{2}{*}{ Gestión Empresarial: misión } & $\mathrm{Si}$ & 11 & \multirow{2}{*}{ 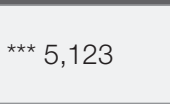 } \\
\hline & No & 102 & \\
\hline \multirow{2}{*}{ Gestión Empresarial: visión } & $\mathrm{Si}$ & 10 & \multirow{2}{*}{$* \star \star 4,661$} \\
\hline & No & 103 & \\
\hline \multirow{2}{*}{ Gestión Empresarial: organigrama } & $\mathrm{Si}$ & 7 & \multirow{2}{*}{${ }^{\star \star \star} 5,247$} \\
\hline & No & 106 & \\
\hline \multirow{2}{*}{$\begin{array}{l}\text { Gestión Empresarial: políticas internas de la } \\
\text { empresa }\end{array}$} & $\mathrm{Si}$ & 8 & \multirow{2}{*}{$* \star \star ~ 10,536$} \\
\hline & No & 105 & \\
\hline \multirow{2}{*}{$\begin{array}{l}\text { Capacidad Productiva: número de unidades } \\
\text { día producidas }\end{array}$} & $<=658$ & 73 & \multirow{2}{*}{ *** 15,270} \\
\hline & $>659$ & 40 & \\
\hline \multirow{2}{*}{$\begin{array}{l}\text { Ha realizado algún cambio o mejora en sus } \\
\text { procesos, productos o sistemas gestión en } \\
\text { los últimos } 2 \text { años }\end{array}$} & $\mathrm{Si}$ & 71 & \multirow[b]{2}{*}{ ** 3,857} \\
\hline & No & 42 & \\
\hline \multirow{2}{*}{$\begin{array}{l}\text { Indique en cuáles ha realizado innovación: } \\
\text { Adquisición de tecnología incorporada a } \\
\text { maquinaria y equipo }\end{array}$} & $\mathrm{Si}$ & 17 & \multirow[b]{2}{*}{ ** 3,839} \\
\hline & No & 96 & \\
\hline \multirow{2}{*}{$\begin{array}{l}\text { Indique en cuáles ha realizado innovación: } \\
\text { Mejoramiento de los sistemas de comer- } \\
\text { cialización }\end{array}$} & $\mathrm{Si}$ & 27 & \multirow{2}{*}{ ** 4,591} \\
\hline & No & 86 & \\
\hline \multirow{2}{*}{$\begin{array}{l}\text { Ha requerido financiamiento adicional duran- } \\
\text { te el tiempo que lleva generando actividades }\end{array}$} & $\mathrm{Si}$ & 49 & \multirow[b]{2}{*}{ ** 4,562} \\
\hline & No & 63 & \\
\hline
\end{tabular}

Nota: significancia *** $1 \% ; * * 5 \% ; * 10 \%$

Como se aprecia en la tabla 4, aquellas microempresas que tienen un desarrollo e implementación de su gestión estratégica empresarial con herramientas como visión, misión, organigrama y políticas internas de manejo de la empresa, tienen una correlación significativa con el promedio de la productividad del empleado; es decir que quienes han logrado desplegar una estrategia empresarial en la organización tienen un promedio de productividad por empleado superior en comparación a aquellas que no lo han desarrollado.

En consecuencia, se evidencia significativamente que aquellas microempresas que han implementado la gestión empresarial tienen un 
incremento promedio de productividad por empleado mes entre USD 583,29 y USD 783,28.

Por otro lado, al analizar la capacidad producida de las microempresas mediante las unidades al día producidas, se evidencia una correlación negativa con respecto al promedio de productividad total de los negocios de panificación; es decir que aquellas microempresas que producen en promedio más de 659 unidades al día tienen un promedio de productividad mes por empleado de USD 934,20 frente a USD 545,79 de aquellas que producen menos de 658 unidades día.

De la misma manera, las microempresas que han realizado cambios o mejoras en sus productos, procesos o sistemas de gestión en los 2 últimos años en adquisición de tecnología incorporada a maquinaria o equipos y en mejoramiento de comercialización, evidencian una media significativa de productividad/mes por empleado que oscila entre USD 587 a USD 895.

De igual forma, las microempresas que han requerido financiamiento adicional durante el tiempo que llevan generando actividades tienen una media significativa de productividad-mes por empleado mayor, que va entre USD 633 a USD 977, en comparación de las que no requirieron financiamiento adicional. Esto tiene una relación directa ya que el recurso que adquiere la empresa se invierte para la compra de maquinaria y ampliación física del negocio, permitiendo un mayor aprovechamiento del desempeño de sus recursos en las microempresas panificadoras.

\section{Conclusiones}

Se aprecia que las microempresas panificadoras tienen una limitada competitividad en factores internos de su propia operación, tales como una gestión administrativa débil, problemas con el mercado, producción y financiamiento, que constituyen elementos que dificultan la gestión e impiden el crecimiento del negocio.

Por otro lado, la capacidad productiva medida por las unidades producidas al día, es un factor que incide en la productividad por empleado, por lo que se ve incrementada considerablemente si las microempresas producen más de 659 unidades. Esto ligado a la capacidad productiva utilizada de las microempresas panificadoras que en promedio tiene un $70 \%$, por lo que se evidencia que las microempresas pueden producir más unidades al día. 
Otro factor que se relaciona con la productividad del empleado en las microempresas panificadoras del Azuay se relaciona con los cambios o mejoras en los procesos, productos o sistemas gestión en los últimos 2 años, especialmente en la adquisición de tecnología incorporada a maquinaria y equipo, y en el mejoramiento de los sistemas de comercialización.

Además, se observa que los empleados de las microempresas panificadoras tienen en promedio 8 años de nivel de educación, por lo que estudios han demostrado que a mayor grado de escolaridad de sus empleados, mayor es la productividad en la empresa; por lo que las microempresas panificadoras tienen un bajo nivel de productividad.

Como se puede apreciar en la caracterización de las microempresas panificadoras en el Azuay, por su estructura organizativa, normativa tributaria y por su restringida gestión empresarial estratégica, hace que tenga una alta limitación para un crecimiento sostenido en el tiempo.

Finalmente, estos factores coadyuvan a que la mayoría de las microempresas se consideren negocios de subsistencia al no disponer de controles operativos para un correcto y oportuno manejo administrativo. Además el hecho de contar con mano de obra no calificada restringe el desarrollo de procesos, productos o sistemas de gestión innovadores. La falta de capacitación del personal en áreas importantes y el bajo nivel de tecnificación de las maquinarias le resta productividad para que los negocios sean rentables y aporten mayores beneficios a la economía del país.

\section{Referencias}

Benavente, J. (2005). Investigación y desarrollo, innovación y productividad: un análisis econométrico a nivel de la firma. Estudios de Economía, 32(1), 39-67.

Bonilla, Y. M., \& Mayorga, J. Z. (2011). Medición de la productividad por el método del valor agregado (MPVA) a las Pymes de familia del sector industria de la ciudad de Bogotá D.C Medición de la productividad por el método del valor agregado (MPVA) a las Pymes de familia del sector industrial. Bogotá, Colombia.

Centro de Comercio Internacional. (2003). Forum de Comercio Internacional. Obtenido de http://www.forumdecomercio.org/La-medici\%C3\%B3n-del-rendimiento/

Centro Europeo de Empresas e Innovación. (2012). Centro Europeo de Empresas e Innovación. Obtenido de http://www.ceeicr.es/innovacion/innovacion-empresarial/

Cohen, M., \& Gabriel, B. (2012). La situación de las PyMEs en América Latina.

Comisión Económica para América Latina y el Caribe (2001). Elementos de Competitividad Sistémica de las PYMEs del Istmo Centroamericano. Naciones Unidas-CEPAL, LC/MEX(L.499), 1-54. 
Córdova, J., \& Naranjo, J. (Abril de 2017). Incidencia de la inversión en innovación en las ventas de productos innovadores. Evidencia empírica en empresas manufactureras de Colombia. Información Tecnológica, 28(2), 153-164.

Crépon, B., Duguet, E., \& Mairessec, J. (1998). Research, innovation and productivity: an econometric analysis at the firm level. Economics of Innovation and New Technology.

Dyer, L., \& Reeves, T. (1995). Human Resource Strategies and Firm Performance: What Do We Know and Where Do We Need to Go? Center for Advanced Human Resource Studies, 1-17.

Etzkowitz, H., \& Leydesdorff, L. (2000). The dynamics of innovation: from National Systems and "Mode 2" to a Triple Helix of university-industry-government relations. Research Policy, 29(2), 109-123. https://doi.org/10.1016/S00487333(99)00055-4

Fairlie, E. (2007). Generación de Valor Agregado en las Pymes a través de la innovación empresarial. Gestión en el tercer milenio. Revista de Investigación de la Facultad de Ciencias Administrativas, Universidad Nacional Mayor San Marcos, 23-27.

Griliches, Z. (1979). Issues in assessing the contribution and development of research to productivity growth. The Bell Journal of Economics, 10(1), 92-116. https:// doi.org/10.2307/3003321

Hidalgo, G., Kamiya, M., \& Reyes, M. (2014). Emprendimientos dinámicos en América Latina (No. $\left.\mathrm{N}^{\circ} 16 / 2014\right)$. Venezuela.

Hoffman, K., Parejo, M., Bessant, J., \& Perren, L. (1998). Small firms, R\&D, technology and innovation in the UK: a literature review. Technovation, 18(1), 39-55. https://doi.org/10.1016/S0166-4972(97)00102-8

Kuznets, S. S. (1966). Modern economic growth : rate, structure, and spread. New Haven: Yale University Press.

Lochon, F. (2014). El salto productivo a la tecnología.

Lööf, H., Heshmati, A., Asplund, R., \& Naas, S. (2001). Innovation and performance in manufacturing industries: a comparison of the Nordic countries. EconStor, SSE/ EFI working paper series in economics and finance(457).

Maldonado Guzmán, G., Martínez Serna, M. del C., Hernández Castorena, O., \& García Pérez de Lema, D. (2011). El impacto de los procesos de producción en el rendimiento de la pyme manufacturera de México: Un estudio empírico. TEC Empresarial, 5(1), 21-30.

Rodríguez, F. X., \& Gómez, L. (2011). Indicadores de calidad y productividad en las empresas. New York.

Rodríguez, L. A., Bernal, M. E., \& Cuervo, L. M. (2012). Teoría y práctica del desarrollo económico local.

Salazar, C. A. (2015). La productividad y competitividad en las PYMES. Quito- Ecuador.

Solís, L. (2005). Procesos de negocios de Pymes insertas en redes colaborativas SMSB negotiating processes inserted into collaborative. Administración de operaciones.

Teece, D. J., Pisano, G., \& Shuen, A. (1997). Dynamic capabilities and strategic management. Strategic Management Journal, 18(7), 509-533. https://doi. org/10.1002/(SICI)1097-0266(199708)18:7<509::AID-SMJ882>3.0.CO;2-Z 
Velarde, E., Araiza, Z., \& García, A. (2014). Factores de la empresa y del empresario y su relación con el éxito económico en la PYMES de la región centro de Coahuila, en México. Revista Internacional de Administración y Finanzas, 7(5), 11-23.

Villegas, C. A. S. (2015). La productividad y competitividad en las PYMES. Quito-Ecuador.

Vossen, R. W. (1998). Relative strengths and weaknesses of small firms in innovation. International Small Business Journal, 16(3), 88-95.

World Bank Group (2014). Doing Business 2014 Measuring Regulatory Quality and Efficiency. https://doi.org/10.1596/978-1-4648-0667-4 